\title{
Special Ball-Homogeneous Spaces
}

\author{
G. Calvaruso and L. Vanhecke
}

Dedicated to the memory of Professor P. Günther

\begin{abstract}
We continue the study of ball-homogeneous Riemannian manifolds, that is, Riemannian spaces such that the volume of all sufficiently small geodesic balls or spheres only depends on the radius. First, we consider the case of locally reducible spaces. Then we treat the three-dimensional case, in particular for Einstein-like metrics and finally, we study conformally flat ball-homogeneous spaces. Our aim is to provide more partial answers to the question whether a ball-homogeneous space is locally homogeneous or not.
\end{abstract}

Keywords: Ball-homogeneous spaces, Einstein-like manifolds, conformally flat spaces AMS subject classification: 53 C 25,53 C 30,53 C 35

\section{Introduction}

$D^{\prime}$ Atri spaces are Riemannian manifolds all of whose local geodesic symmetries are volume-preserving (up to sign). These spaces are natural generalizations of locally symmetric manifolds. Many examples have been discovered and their geometry has been treated in several papers. We refer to [14] for a rather complete survey and for further references. Up to now there are no examples known which are not locally homogeneous and so, it is still an open problem whether there are D' Atri spaces which are not locally homogeneous.

In [11] it is shown that each D' Atri space is ball-homogeneous, that is, the volume of every sufficiently small geodesic sphere or ball does not depend on the center of the ball but is only a function of the radius [15]. Clearly, locally homogeneous spaces are trivial examples but although the class of ball-homogeneous spaces is much broader than that of D' Atri spaces, also here all the known examples are again locally homogeneous. This fact raised the question: Are ball-homogeneous spaces necessarily locally homogeneous? Several aspects of the geometry of ball-homogeneous spaces have been treated in $[4,5$, $7,15,20]$ but the above question remains open and only partial positive answers could be provided.

In this paper we continue this study and look for properties which hold for D' Atri spaces and which can be extended to the broader class of ball-homogeneous spaces. In

G. Calvaruso: Katholieke Universiteit Leuven, Department of Mathematics, Celestijnenlaan 200B, 3001 Leuven, Belgium. Supported by grant decr. n. 1106 of 26/09/1995 of Università degli Studi di Lecce (Italy).

L. Vanhecke: Katholieke Universiteit Leuven, Department of Mathematics, Celestijnenlaan 200B, 3001 Leuven, Belgium. 
Section 2 we use a mean-value formula to prove that an analytic Riemannian product is ball-homogeneous if and only if each factor is ball-homogeneous. Then, in Section 3, we consider the above question for three-dimensional spaces. It turns out that already here it seems to be difficult to obtain a complete answer since we can only give a positive solution when there are at most two distinct eigenvalues for the Ricci operator. This contrasts to the case of D' Atri spaces where it has been shown that every threedimensional D' Atri space is locally isometric to a naturally reductive space and hence, is locally homogeneous. This leads in Section 4 to the consideration of special threedimensional ball-homogeneous spaces, namely those equipped with Einstein-like metrics [9]. Here we shall show that a three-dimensional ball-homogeneous space $\left(M^{3}, g\right)$ whose Ricci tensor is of Codazzi type (and hence, $\left(M^{3}, g\right)$ is conformally flat) is necessarily locally symmetric while an $\left(M^{3}, g\right)$ is locally isometric to a naturally reductive space if and only if it is ball-homogeneous and has cyclic-parallel Ricci tensor. Finally, in Section 5 we treat conformally flat ball-homogeneous spaces and prove that such manifolds are locally symmetric if the Ricci operator has at most three distinct eigenvalues, the general case remaining open. Our proof uses the following extension of a result proved by $\mathrm{H}$. Takagi [19] for homogeneous spaces: Every curvature homogeneous conformally flat space is necessarily locally symmetric.

We thank F. Prüfer for useful discussions about the use of mean-value formulas in Section 2.

\section{Ball-homogeneous Riemannian products}

Let $(M, g)$ be an $n$-dimensional, connected, smooth Riemannian manifold with Levi Civita connection $\nabla$. We denote by $R$ its Riemannian curvature tensor taken with the sign convention $R_{X Y}=\nabla_{[X, Y]}-\left[\nabla_{X}, \nabla_{Y}\right]$ for all smooth vector fields $X, Y$ on $M$. Further, $\varrho$ and $\tau$ denote its Ricci tensor and scalar curvature, respectively. Let $\Delta$ be the Laplacian on $(M, g)$.

Next, let $m \in M$ and denote by $\exp _{m}$ the exponential map centered at $m$. For $r<i(m)$, the injectivity radius at $m$, we denote by $G_{m}(r)=\exp _{m} S_{m}(r)$ the geodesic sphere with center $m$ and radius $r$ where $S_{m}(r)$ is the sphere of center 0 and radius $r$ in $T_{m} M$. Let $B_{m}(r)$ be the corresponding geodesic ball and denote by $V_{m}(r)$ its volume, that is,

$$
V_{m}(r)=\int_{0}^{r} \int_{S^{n-1}(1)} t^{n-1} \theta_{m}\left(\exp _{m} t u\right) d u d t
$$

where $u$ is a unit vector of $T_{m} M$ and $\theta_{m}$ is the volume density function $\theta_{m}=\left(\operatorname{det}\left(g_{i j}\right)\right)^{\frac{1}{2}}$ of $\exp _{m}$.

The Riemannian manifold $(M, g)$ is said to be ball-homogeneous if $V_{m}(r)$ only depends on $r$ [15]. We prove the following extension of a result for 'D' Atri spaces.

Theorem 2.1. Let $(M, g)=\left(M_{1}, g_{1}\right) \times \cdots \times\left(M_{r}, g_{r}\right)$ be an analytic Riemannian product. Then $(M, g)$ is ball-homogeneous if and only if each factor $\left(M_{i}, g_{i}\right)(i=1, \ldots, r)$ is ball-homogeneous. 
Proof. Obviously, it is enough to restrict to the case where $r=2$. Let $\tilde{\Delta}^{(k)}$ be the Euclidean Laplacian of order $k$ defined for a function $\varphi$ by

$$
\tilde{\Delta}^{(k)}[\varphi](m)=\frac{1}{(2 k) !} \sum_{i_{1} \ldots i_{2 k}=1}^{n} \sum_{\sigma}\left\{\delta_{i_{\sigma(1)} i_{\sigma(2)}} \cdots \delta_{i_{\sigma(2 k-1)} i_{\sigma(2 k)}} \nabla_{i_{1} \ldots i_{2 k}}^{2 k} \varphi\right\}(m)
$$

Here $\left\{e_{1}, \ldots, e_{n}\right\}$ is an arbitrary local orthonormal basis, $\nabla_{i_{1} \ldots i_{k}}^{k}=\nabla_{e_{i_{1}} \ldots e_{i_{k}}}^{k}, \delta_{i j}$ denotes the Kronecker symbol, and the summation is made over all permutations $\sigma$ of the set $\{1, \ldots, 2 k\}$. These Euclidean Laplacians are globally defined differential operators of order $2 k$. See [14] for details and references. By using the generalized Pizetti formula for mean-value operators, we may express the volume $m_{r}=\frac{d V_{m}(r)}{d r}$ of a geodesic sphere on an analytic $(M, g)$ by means of these Euclidean Laplacians. Explicitly, we have

$$
m_{r}=2 \pi^{\frac{n}{2}} r^{n-1} \sum_{k=0}^{+\infty}\left(\frac{r}{2}\right)^{2 k} \frac{1}{k ! \Gamma\left(\frac{n}{2}+k\right)} D_{M}^{2 k}(m)
$$

where $D_{M}^{2 k}(m)=\tilde{\Delta}^{(k)}\left[\theta_{m}\right](m)$. Here, $D_{M}^{0}(m)=1$. Hence, $(M, g)$ is ball-homogeneous if and only if $D_{M}^{2 k}$ is globally constant for each $k \in \mathbb{N}$.

Next, let $(M, g)=\left(M_{1}, g_{1}\right) \times\left(M_{2}, g_{2}\right)$. Then we have

$$
\tilde{\Delta}_{M}^{(k)}=\sum_{\lambda=0}^{k}\left(\begin{array}{l}
k \\
\lambda
\end{array}\right) \tilde{\Delta}_{M_{1}}^{(\lambda)} \tilde{\Delta}_{M_{2}}^{(k-\lambda)}
$$

and $\theta_{M}=\theta_{M_{1}} \cdot \theta_{M_{2}}$. So, for $m=\left(m_{1}, m_{2}\right)$, we have

$$
D_{M}^{2 k}(m)=\sum_{\lambda=0}^{k}\left(\begin{array}{l}
k \\
\lambda
\end{array}\right) D_{M_{1}}^{2 \lambda}\left(m_{1}\right) D_{M_{2}}^{2 k-2 \lambda}\left(m_{2}\right)
$$

The theorem follows now easily from this formula. Indeed, let $\left(M_{1}, g_{1}\right)$ and $\left(M_{2}, g_{2}\right)$ be ball-homogeneous. Then $D_{M_{1}}^{2 p}$ and $D_{M_{2}}^{2 q}$ are globally constant for all $p, q, \in \mathbb{N}$. Hence, $D_{M}^{2 p}$ is also globally constant. Conversely, let $(M, g)$ be a ball-homogeneous space. We have, for $s \geq 1$,

$$
D_{M}^{2 s}(m)=D_{M_{1}}^{2 s}\left(m_{1}\right)+D_{M_{2}}^{2 s}\left(m_{2}\right)+\sum_{\lambda=1}^{s-1}\left(\begin{array}{l}
s \\
\lambda
\end{array}\right) D_{M_{1}}^{2 \lambda}\left(m_{1}\right) D_{M_{2}}^{2 s-2 \lambda}\left(m_{2}\right)
$$

An induction procedure yields $D_{M_{1}}^{2 s}\left(m_{1}\right)+D_{M_{2}}^{2 s}\left(m_{2}\right)=$ const and hence, $D_{M_{1}}^{2 p}$ and $D_{M_{2}}^{2 p}$ are constant for all $p \in \mathbb{N}$, which proves the required result

Remark. When $(M, g)$ is an analytic, complete Riemannian product, then the result may be obtained by using the recursion formula given in [8]. 


\section{Three-dimensional ball-homogeneous spaces}

The condition of ball-homogeneity implies the constancy of an infinite number of scalar curvature invariants. Indeed, when a Riemannian manifold $(M, g)$ is ball-homogeneous, then $\frac{d^{k} V_{m}(r)}{d r^{k}}(m)$ must be constant on $M$ for all $k \in \mathbb{N}$. These derivatives may be expressed by using scalar curvature invariants but the explicit formulas are not known for general $k$. Nevertheless, we have the following formula for $\operatorname{dim} M=n[10]$ :

$$
V_{m}(r)=\omega r^{n}\left\{1+A(m) r^{2}+B_{j}(m) r^{4}+C(m) r^{6}+O\left(r^{8}\right)\right\}
$$

where

$$
\omega=\frac{\pi^{\frac{n}{2}}}{\left(\frac{n}{2}\right) !}
$$

and

$$
\begin{aligned}
A= & -\frac{\tau}{6(n+2)}, \\
B= & \frac{1}{360(n+2)(n+4)}\left(-3\|R\|^{2}+8\|\varrho\|^{2}+5 \tau^{2}-18 \tau \Delta \tau\right) \\
C= & \frac{1}{720(n+2)(n+4)(n+6)}\left\{-\frac{5}{9} \tau^{3}-\frac{8}{3} \tau\|\varrho\|^{2}+\tau\|R\|^{2}\right. \\
& +\frac{64}{63} \varrho+\frac{64}{21} g(\varrho \otimes \varrho, \bar{R})+\frac{32}{7} g(\varrho, \dot{R})-\frac{110}{63} \check{R}-\frac{200}{63} \overline{\bar{R}} \\
& +\frac{45}{7}\|\nabla \tau\|^{2}+\frac{45}{14}\|\nabla \varrho\|^{2}+\frac{45}{7} \alpha(\varrho)-\frac{45}{14}\|\nabla R\|^{2}+6 \tau \Delta \tau \\
& \left.+\frac{48}{7} g(\Delta \varrho, \varrho)+\frac{54}{7} g\left(\nabla^{2} \tau, \varrho\right)-\frac{30}{7} g(\Delta R, R)-\frac{45}{7} \Delta^{2} \tau\right\}
\end{aligned}
$$

Here, we have with respect to an orthonormal basis:

$$
\begin{array}{ll}
\|\varrho\|^{2}=\sum_{i, j} \varrho_{i j}^{2}, & \|R\|^{2}=\sum_{i, j, k, l} R_{i j k l}^{2}, \\
\check{\varrho}=\sum_{i, j, k} \varrho_{i j} \varrho_{j k} \varrho_{k i}, & g(\varrho \otimes \varrho, \bar{R})=\sum_{i, j, k, l} \varrho_{i j} \varrho_{k l} R_{i k j l}, \\
\check{R}=\sum_{i, j, k, l, p, q} R_{i j k l} R_{k l p q} R_{p q i j}, & \check{R}=\sum_{i, j, k, l, p, q} R_{i k j l} R_{k p l q} R_{p i q j}, \\
g(\varrho, \dot{R})=\sum_{i, j, p, q, r} \varrho_{i j} R_{i p q r} R_{j p q r}, & \alpha(\varrho)=\sum_{i, j, k} \nabla_{i} \varrho_{j k} \nabla_{k} \varrho_{i j} .
\end{array}
$$

For a three-dimensional Riemannian manifold; the vanishing of the Weyl tensor 
yields the following relations [10]:

$$
\left.\begin{array}{rl}
\|R\|^{2} & =4\|\varrho\|^{2}-\tau^{2} \\
g(\varrho \otimes \varrho, \bar{R}) & =\frac{5}{2} \tau\|\varrho\|^{2}-\frac{1}{2} \tau^{3}-2 \check{\varrho} \\
g(\varrho, \dot{R}) & =4 \tau\|\varrho\|^{2}-\tau^{3}-2 \check{\varrho} \\
\check{R} & =12 \tau\|\varrho\|^{2}-3 \tau^{3}-8 \check{\varrho} \\
\check{R} & =\frac{3}{2} \tau\|\varrho\|^{2}-\frac{1}{4} \tau^{3}-2 \check{\varrho} \\
\|\nabla R\|^{2} & =4\|\nabla \varrho\|^{2}-\|\nabla \tau\|^{2} \\
g(\Delta R, R) & =4 g(\Delta \varrho, \varrho)-\tau \Delta \tau .
\end{array}\right\}
$$

Further, we note that we always have

$$
\left.\begin{array}{l}
\frac{1}{2} \Delta\|R\|^{2}=g(\Delta R, R)+\|\nabla R\|^{2} . \\
\frac{1}{2} \Delta\|\varrho\|^{2}=g(\Delta \varrho, \varrho)+\|\nabla \varrho\|^{2} .
\end{array}\right\}
$$

Using these formulas, we then get at once

Proposition 3.1. Let $(M, g)$ be a three-dimensional ball-homogeneous space. Then

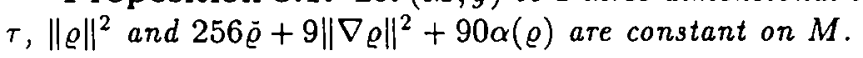

Now, we prove

Theorem 3.2. A three-dimensional ball-homogeneous space $(M, g)$ with at most two distinct Ricci eigenvalues is locally homogeneous.

Proof. Let $W$ be the dense open subset of $M$ where the multiplicities of the eigenvalues $\varrho_{1}, \varrho_{2}$ and $\varrho_{3}$ of the Ricci operator are locally constant. So, let $p \in W$ and consider a neighborhood $U$ of $p$ where these multiplicities are constant. In what follows we shall show that all the scalar curvature invariants are constant on $U$. A continuity argument then shows that these invariants are constant on $M$. Then it follows from the main result in [18] (see also [20]) that $(M, g)$ is locally homogeneous.

So, in what follows we concentrate on $U$ and consider the following two cases: $\varrho_{1}=\varrho_{2}=\varrho_{3}$ and $\varrho_{1}=\varrho_{2} \neq \varrho_{3}$. For the first case it follows that $U$ is Einsteinian and hence locally symmetric. For the second case and since $2 \varrho_{1}+\varrho_{3}=\tau, 2 \varrho_{1}^{2}+\varrho_{3}^{2}=\|\varrho\|^{2}$, it follows from Proposition 3.1 that $\varrho_{1}$ and $\varrho_{3}$ are constant on $U$. We consider the cases $\varrho_{3} \neq 0$ and $\varrho_{3}=0$.

a) Case $\varrho_{3} \neq 0$ : This case has been treated in [12] where it is shown that there exists a local orthonormal frame $\left\{E_{1}, E_{2}, E_{3}\right\}$ with dual coframe $\left\{\omega^{1}, \omega^{2}, \omega^{3}\right\}$ such that

$$
\begin{aligned}
\nabla \varrho= & \left(\varrho_{3}-\varrho_{1}\right)\left\{\left(a \omega^{1}+b \omega^{2}\right) \otimes\left(\omega^{1} \otimes \omega^{3}+\omega^{3} \otimes \omega^{1}\right)+\right. \\
& \left.+\left(c \omega^{1}+e \omega^{2}\right) \otimes\left(\omega^{2} \otimes \omega^{3}+\omega^{3} \otimes \omega^{2}\right)\right\}
\end{aligned}
$$


This yields

$$
\left.\begin{array}{rl}
\|\nabla \varrho\|^{2} & =2\left(\varrho_{1}-\varrho_{3}\right)^{2}\left(a^{2}+b^{2}+c^{2}+e^{2}\right) \\
\alpha(\varrho) & =\left(\varrho_{1}-\varrho_{3}\right)^{2}\left(a^{2}+e^{2}+2 b c\right) .
\end{array}\right\}
$$

Further, since $\nabla_{E_{3}} \tau=0$, we have $a+e=0$. Moreover, it follows from Propositions 5.6 and 7.2 in [12] that

$$
\|\dot{\nabla} \varrho\|^{2}=2\left(\varrho_{1}-\varrho_{3}\right)^{2}\left(h^{2}-\varrho_{3}\right)
$$

where $h=b-c$. So $h^{2}-\varrho_{3}=2 a^{2}+b^{2}+c^{2}$, that is, $-\varrho_{3}=2 a^{2}+2 b c$. Hence, we have

$$
\left.\begin{array}{rl}
\|\nabla \varrho\|^{2} & =2\left(\varrho_{1}-\varrho_{3}\right)^{2}\left(h^{2}-\varrho_{3}\right) \\
\alpha(\varrho) & =-\left(\varrho_{1}-\varrho_{3}\right)^{2} \varrho_{3} .
\end{array}\right\}
$$

Using Proposition 3.1 and (7), we obtain that $h$ is constant and this implies that $U$ is locally homogeneous [12: Proposition 7.3], from which it follows that all scalar curvature invariants are constant on $U$.

b) Case $\varrho_{3}=0$ : The condition $\varrho_{3}=0$ implies that $(U, g)$ is semi-symmetric, that is, $R_{X Y} \cdot R=0$, and has been treated in detail in [13] (see also [12: Theorem A] and $[3])$. There it is shown that when $(U, g)$ is not locally homogeneous, there exists an open dense subset $V$ of $U$ such that in a neighborhood of each point $p \in V, U$ is isometric to the space $(N, \tilde{g})$ where $N$ is a domain of $\mathbb{R}^{3}$ and $\tilde{g}$ is the Riemannian metric given by $\tilde{g}=\sum_{i=1}^{3} \omega_{i} \otimes \omega_{i}$ and

$$
\left.\begin{array}{l}
\omega^{0}=f\left(x_{0}, x_{1}\right) d x_{1} \\
\omega^{1}=d x_{1}-x_{2} d x_{0} \\
\omega^{2}=d x_{2}+x_{1} d x_{0}
\end{array}\right\}
$$

Here $\tau=-2 \frac{f_{x_{1} x_{1}}^{\prime \prime}}{f} \neq 0$ and hence, we have

$$
f\left(x_{0}, x_{1}\right)= \begin{cases}a\left(x_{0}\right) e^{k x_{1}}+b\left(x_{0}\right) e^{-k x_{1}} & \text { for } \tau=-2 k^{2}<0 \\ a\left(x_{0}\right) \cos \left(k x_{1}\right)+b\left(x_{0}\right) \sin \left(k x_{1}\right) & \text { for } \tau=2 k^{2}>0 .\end{cases}
$$

Using the structure equations of Cartan, we get

$$
\left.\begin{array}{rl}
\omega_{1}^{0} & =f^{-1} f_{x_{1}}^{\prime} \omega^{0} \\
\omega_{2}^{0} & =0 \\
\omega_{2}^{1} & =-f^{-1} \omega^{0} \\
\omega_{j}^{i}+\omega_{i}^{j} & =0 \quad(i, j=0,1,2) .
\end{array}\right\}
$$

A straightforward computation then yields

$$
\left.\begin{array}{c}
\varrho=\frac{\tau}{2}\left(\omega^{0} \otimes \omega^{0}+\omega^{1} \otimes \omega^{1}\right) \\
\nabla_{X \varrho}=-\frac{\tau}{2} f^{-1} \omega^{0}(X)\left(\omega^{1} \otimes \omega^{2}+\omega^{2} \otimes \omega^{1}\right)
\end{array}\right\}
$$


and hence,

$$
\left.\begin{array}{rl}
\|\nabla \varrho\|^{2} & =\frac{\tau^{2}}{2} f^{-2} \\
\alpha(\varrho) & =0 .
\end{array}\right\}
$$

Using (9), Proposition 3.1 again implies that $\|\nabla \varrho\|^{2}$, and hence $f$, must be constant but this contradicts $\tau \neq 0$. So, also in this case $(U, g)$ must be locally homogeneous. This completes the proof of the theorem

Remarks. (A) Let $\left(M^{3}, g, \xi, \eta, \varphi\right)$ be an almost contact metric manifold [2]. Then $\left(M^{3}, g\right)$ is said to be $\eta$-Einsteinian if

$$
\varrho=a g+b \eta \otimes \eta
$$

In this case $\varrho_{1}=\varrho_{2}=a, \varrho_{3}=a+b$. Hence, the Ricci operator has at most two distinct eigenvalues. Thus we have: $A$ ball-homogeneous $\eta$-Einsteinian almost contact metric manifold $\left(M^{3}, g, \xi, \eta, \varphi\right)$ is locally homogeneous. We refer to [17] for a treatment of three-dimensional homogeneous contact metric spaces. Note that since $\tau$ has to be constant, any threc-dimensional ball-homogeneous Sasakian manifold is locally $\varphi$ symmetric and hence locally homogeneous. See, for example; $[7,14]$.

Note that the same result holds for any $\left(M^{3}, g\right)$ with a Ricci tensor which is expressible in the form given above.

(B) In [6], it has been proved that a three-dimensional Riemannian manifold is pseudo-symmetric if and only if the Ricci operator has at most two distinct eigenvalues. So, Theorem 3.2 may be reformulated as follows: A three-dimensional ball-homogeneous pseudo-symmetric Riemannian manifold is locally homogeneous.

\section{Ball-homogeneous Einstein-like spaces of dimension three}

In Section 3 we could only provide partial answers (for three-dimensional Riemannian manifolds) to the fundamental problem stated in the Introduction. For that reason we shall now continue our study of three-dimensional ball-homogeneous spaces $(M, g)$ when they are equipped with special metrics and we concentrate on Einstein-like manifolds. These spaces have been introduced in [9]. Here, we shall consider $\left(M^{3}, g\right)$ such that the Ricci tensor $\varrho$ is a Codazzi tensor, that is, $\left(\nabla_{X} \varrho\right)(Y, Z)=\left(\nabla_{Y} \varrho\right)(X, Z)$, or is cyclicparallel, that is, $\left(\nabla_{X} \varrho\right)(Y, Z)+\left(\nabla_{Y} \varrho\right)(Z, X)+\left(\nabla_{Z} \varrho\right)(X, Y)=0$. This last condition means that $\varrho$ is a Killing tensor. Note that in both cases $\tau$ is necessarily constant.

As it is well-known, when $\tau$ is constant, $\left(M^{3}, g\right)$ has a Codazzi-Ricci tensor if and only if it is conformally flat. For that reason we first state a useful theorem about conformally flat spaces. In [19] it is proved that any conformally flat locally homogeneous space is locally symmetric. Using the same proof, this result can be extended to the broader class of curvature homogeneous'spaces. Note that a Riemanian manifold $(M, g)$ is said to be curvature homogeneous if for each pair of points $p, q \in M$ there exists a linear isometry $\varphi: T_{p} M \rightarrow T_{q} M$ such that $\varphi^{*} R_{q}=R_{p}$. See [3] for a survey and further references. So, we have 
Proposition 4.1. A curvature homogeneous conformally flat Riemannian manifold is locally symmetric.

Clearly, a three-dimensional Riemannian manifold is curvature homogeneous if and only if the eigenvalues of the Ricci operator are constant.

Now we are ready to prove our first result.

Theorem 4.2. $\left(M^{3}, g\right)$ is locally symmetric if and only if it is ball-homogeneous and its Ricci tensor is a Codazzi tensor.

Proof. The "only if" part is trivial. So, we consider the "if" part. Since

$$
\sum_{i, j, k}\left(\nabla_{i} \varrho_{j k}-\nabla_{j} \varrho_{i k}\right)^{2}=2\left(\|\nabla \varrho\|^{2}-\alpha(\varrho)\right),
$$

we get that $\varrho$ is a Codazzi tensor if and only if

$$
\|\nabla \varrho\|^{2}=\alpha(\varrho)
$$

Moreover, we have [10: Formula (2.16)]

$$
\sum_{i, j, k} \varrho_{j k} \nabla_{i j}^{2} \varrho_{i k}=\frac{1}{2} g\left(\nabla^{2} \tau, \varrho\right)+\check{\varrho}-g(\varrho \otimes \varrho, \bar{R}) .
$$

Next, since $\varrho$ is of Codazzi type, we get at once

$$
\sum_{i, j, k} \varrho_{j k} \nabla_{i j}^{2} \varrho_{i k}=\frac{1}{2} \Delta\|\varrho\|^{2}-\|\nabla \varrho\|^{2} .
$$

Using now that $\tau$ and $\|\varrho\|^{2}$ are constant, we get from (11), (12) and (5)

$$
\|\nabla \varrho\|^{2}=\frac{5}{2} \tau\|\varrho\|^{2}-\frac{1}{6} \tau^{3}-3 \check{\varrho} .
$$

Hence, Proposition $3.1,(10)$ and (13) yield that $\check{\varrho}$ is constant. From this, together with $\tau=$ const and $\|\varrho\|^{2}=$ const, we obtain that the eigenvalues of the Ricci operator are constant. So, $\left(M^{3}, g\right)$ is curvature homogeneous. Then the result follows from Proposition 4.1

This implies

Corollary 4.3. $\left(M^{3}, g\right)$ is locally symmetric if and only if it is ball-homogeneous and conformally flat.

Next, we turn to the case of a cyclic-parallel Ricci tensor. We mention the following useful results.

Proposition 4.4 (see [1]). A Riemannian manifold $\left(M^{3}, g\right)$ is locally isometric to a naturally reductive homogeneous space if and only if it is locally homogeneous and has a cyclic-parallel Ricci tensor.

Proposition 4.5 (see [21]). Let $\left(\dot{M}^{3}, g\right)$ be a Riemannian manifold with three distinct and constant eigenvalues of the Ricci operator. If $\sum_{a, b} \varrho_{a b} \nabla_{b} \varrho_{i a}=0$, then $\left(M^{3}, g\right)$ is locally homogeneous.

Now we prove 
Theorem 4.6. $\left(M^{3}, g\right)$ is locally isometric to a naturally reductive homogeneous space if and only if it is ball-homogeneous and has cyclic-parallel Ricci tensor.

Proof. First, let $\left(M^{3}, g\right)$ be locally isometric to a naturally reductive homogeneous space. Then it is clearly ball-homogeneous and moreover, $\varrho$ is cyclic-parallel (see, for example, [14]). To prove the converse, we shall first show that $\left(M^{3}, g\right)$ is a curvature homogeneous space. Since $\varrho$ is cyclic-parallel, we have

$$
2 \alpha(\varrho)=-\|\nabla \varrho\|^{2}
$$

and moreover,

$$
\sum_{i, j, k} \varrho_{j k} \nabla_{i j}^{2} \varrho_{i k}=-\frac{1}{4} \Delta\|\varrho\|^{2}+\frac{1}{2}\|\nabla \varrho\|^{2} .
$$

Since $\|\nabla \varrho\|^{2}$ is constant, we get from (5), (1 $\left.\overline{1}\right)$ and $(\overline{1} 5)$

$$
\|\nabla \varrho\|^{2}=6 \check{\varrho}-5 \tau\|\varrho\|^{2}+\tau^{3} .
$$

Hence, the constancy of $\tau$ and $\|\varrho\|^{2}$, together with (14), (16) and Proposition 3.1 imply that $\check{\varrho}$ is constant. So, the eigenvalues of $\varrho$ are constant and $\left(M^{3}, g\right)$ is curvature homogeneous.

If $\varrho$ has less than three distinct eigenvalues, the required result follows from Theorem 3.2 and Proposition 4.4.

If $\varrho$ has three distinct eigenvalues, we use Propositions 4.4 and 4.5 . Indeed, since $\varrho$ is cyclic-parallel, we have

$$
2 \sum_{a, b} \varrho_{a b} \nabla_{b} \varrho_{i a}=-\sum_{a, b} \varrho_{a b} \nabla_{i} \varrho_{a b}=-\frac{1}{2} \nabla_{i}\|\varrho\|^{2}=\cdot 0
$$

and the result follows

\section{Conformally flat ball-homogeneous spaces}

Based on the method used and the results mentioned in Section 4, we focus now on $n$-dimensional manifolds $(n>2)$ with vanishing Weyl tensor $W$, that is, on conformally flat spaces when $n \geq 4$. In this case we have, with respect to an arbitrary orthonormal basis,

$$
\begin{aligned}
R_{i j k h}= & \frac{1}{n-2}\left(\delta_{i k} \varrho_{j h}+\delta_{j h} \varrho_{i k}-\delta_{i h} \varrho_{j k}-\delta_{j k} \varrho_{i h}\right) \\
& -\frac{\tau}{(n-1)(n-2)}\left(\delta_{i k} \delta_{j h}-\delta_{i h} \delta_{j k}\right)
\end{aligned}
$$

and a straightforward computation yields the following relations for the scalar curvature invariants mentioned in (3), (4) (see also [16]):

$$
\|R\|^{2}=\frac{4}{n-2}\|\varrho\|^{2}-\frac{2}{(n-1)(n-2)} \tau^{2},
$$




$$
\begin{aligned}
\|\nabla R\|^{2} & =\frac{4}{n-2}\|\nabla \varrho\|^{2}-\frac{2}{(n-1)(n-2)}\|\nabla \tau\|^{2}, \\
\check{R} & =\frac{24}{(n-1)(n-2)^{3}} \tau\|\varrho\|^{2}-\frac{4 n}{(n-1)^{2}(n-2)^{3}} \tau^{3}+\frac{8(n-4)}{(n-2)^{3}} \check{\varrho}, \\
\check{R} & =\frac{3 n^{2}-6 n-6}{(n-1)(n-2)^{3}} \tau\|\varrho\|^{2}-\frac{2 n^{2}-7 n+4}{(n-1)^{2}(n-2)^{3}} \tau^{3}+\frac{2(8-3 n)}{(n-2)^{3}} \check{\varrho} \\
g(\varrho \otimes \varrho, \bar{R}) & =\frac{2 n-1}{(n-1)(n-2)} \tau\|\varrho\|^{2}-\frac{1}{(n-1)(n-2)} \tau^{3}-\frac{2}{n-2} \check{\varrho}, \\
g(\varrho, \dot{R}) & =\frac{2(n+1)}{(n-1)(n-2)^{2}} \tau\|\varrho\|^{2}-\frac{2 .}{(n-1)(n-2)^{2}} \tau^{3}+\frac{2(n-4)}{(n-2)^{2}} \check{\varrho} .
\end{aligned}
$$

Now we prove a generalization of Corollary 4.3.

Theorem 5.1. An $n$-dimensional conformally flat ball-homogeneous space $(M, g)$ with at most three distinct Ricci roots is locally symmmetric.

Proof. Since $(M, g)$ is ball-homogeneous, it follows from (2), (3) and (17) that $\tau$ and $\|\varrho\|^{2}$ are constant. This, (6), (17) - (22) and the constancy of $C$ in (4) then yield

$$
k(n) \check{\varrho}+\frac{3(54-17 n)}{14(n-2)}\|\nabla \varrho\|^{2}+\frac{45}{7} \alpha(\varrho)=\text { const }
$$

where

$$
k(n)=\frac{64\left(n^{3}+9 n^{2}-61 n+93\right)}{63(n-2)^{3}}
$$

Further, it is well-known that $W=0$ implies

$$
\nabla_{k} \varrho_{i j}-\nabla_{j} \varrho_{i k}=\frac{1}{2(n-1)}\left(g_{i j} \nabla_{k} \tau-g_{i k} \nabla_{j} \tau\right)
$$

and hence, since $\tau$ is constant, $\varrho$ is a Codazzi tensor. This implies that (10) holds (note that (10) - (12) hold for general dimension $n$ ). and so, we have

$$
k(n) \check{\varrho}+\frac{3(13 n-6)}{14(n-2)}\|\nabla \varrho\|^{2}=\text { const } .
$$

Further, using (11), (12) and (21), we obtain

$$
\|\nabla \varrho\|^{2}=\frac{2 n-1}{(n-1)(n-2)} \tau\|\varrho\|^{2}-\frac{1}{(n-1)(n-2)} \tau^{3}-\frac{n}{n-2} \check{\varrho}
$$

and with this we get from (25)

$$
\bar{k}(n)\|\nabla \varrho\|^{2}=\mathrm{const}
$$

where

$$
\bar{k}(n)=223 n^{3}-2016 n^{2}+8132 n-11904 .
$$


Since $\bar{k}(3)=369$ and $\bar{k}$ is an increasing function, $\bar{k}(n) \neq 0$ for any $n \geq 3$. Then (26) implies $\|\nabla \varrho\|^{2}=$ const and so, also $\check{\varrho}$ is constant. Consider again the open dense subset $U$ on which the multiplicities of the eigenvalues are locally constant. Let $p \in U$ and let $V$ be a neighborhood of $p$ in $U$ where the multiplicities are constant. Then it follows from the results above, and the fact that there are at most three distinct Ricci roots that the eigenvalues are constant on $V$ and hence $(M, g)$ is curvature homogeneous on $V$. Proposition 4.1 then implies that $(M, g)$ is locally symmetric on $U$ and hence, by a continuity argument, $(M, g)$ is locally symmetric

Remark. Remark (B) of Section 3 can be extended to $n$-dimensional manifolds with vanishing Weyl tensor [6]. Hence, we have: An $n$-dimensional ball-homogeneous pseudo-symmetric space which is conformally flat is locally homogeneous.

\section{References}

[1] Abbena, E., Garbiero, S. and L. Vanhecke: Einstein-like metrics on three-dimensional Riemannian homogeneous manifolds. Simon Stevin 66 (1992), 173 - 182.

[2] Blair, D. E.: Contact Manifolds in Riemannian Geometry. Lect. Notes Math. 509 (1976), $1-146$.

[3] Boeckx, E., Kowalski, O. and L. Vanhecke: Riemannian Manifolds of Conullity Two. Singapore: World Sci. Publ. 1996.

[4] Calvaruso, G., Tondeur, Ph. and L. Vanhecke: Four-dimensional ball-homogeneous and C-spaces. Beitr. Alg. Geom. (to appear).

[5] Calvaruso, G. and L. Vanhecke: Semi-symmetric ball-homogeneous spaces and a volume conjecture. Bull. Austral. Math. Soc. (to appear).

[6] Deprez, J., Deszcz, R. and L. Verstraelen: Examples of pseudo-symmetric conformally flat warped products. Chinese J. Math. 17 (1989), $51-65$.

[7] García-Rio, E. and L. Vanhecke: Five-dimensional $\varphi$-symmetric spaces. Balkan J. Geom. Appl. (to appear).

[8] Gray, A.: Geodesic balls in Riemannian product manifolds. In: Differential Geometry and Relativity (Math. Phys. and Appl. Math: Vol. 3; eds.: M. Cahen and M. Flato). Dordrecht: Reidel Publ. Co. 1976, pp. $63-66$.

[9] Gray, A.: Einstein-like manifolds which are not Einstein. Geom. Dedicata 7 (1978), 259 -280 .

[10] Gray, A. and L. Vanhecke: Riemannian geometry as determined by the volumes of small geodesic balls. Acta Math. 142 (1979), 157 - 198.

[11] Günther, P. and F. Prüfer: D' Atri spaces are ball-homogeneous. Preprint 1995.

[12] Kowalski, O.: A classification of Riemannian 3-manifolds with constant principal Ricci curvatures $\varrho_{1}=\varrho_{2} \neq \varrho_{3}$. Nagoya Math. J. 132 (1993), $1-36$.

[13] Kowalski, O.: An explicit classification of 3-dimensional Riemannian spaces satisfying $R_{X Y} \cdot R=0$. Czechoslovak Math. J. 46(121) (1996), $427-474$.

[14] Kowalski, O., Prüfer, F. and L. Vanhecke: D'Atri spaces. In: Topics in Geometry: In Memory of Joseph D'Atri (Progr. Nonlin. Diff. Equ.: Vol. 20; ed.: S. Gindikin). Boston - Basel - Berlin: Birkhäuser Verlag 1996, pp. $241-284$. 
[15] Kowalski, O. and L. Vanhecke: Ball-homogeneous and disk-homogeneous Riemannian manifolds. Math. Z. 180 (1982), $429-444$.

[16] Perrone, D.: Varietà conformemente piatte e geometria spettrale. Riv. Mat. Univ. Parma 4 (1982), 317 - 330.

[17] Perrone, D.: Homogeneous contact Riemannian three-manifolds. Preprint 1997.

[18] Prüfer, F., Tricerri, F. and L. Vanhecke: Curvature invariants, differential operators and local homogeneity. Trans. Amer. Math. Soc. 348 (1996), 4643 - 4652.

[19] Takagi, H.: Conformally flat Riemannian manifolds admitting a transitive group of isometries. Tôhoku Math. J. 27 (1975), 103 - 110.

[20] Vanhecke, L.: Scalar curvalure invariants and local homogeneity. In: Proc. Workshop on Differential Geometry and Topology (dedicated to the memory of Franco Tricerri), Palermo 1996 (to appear).

[21] Yamato, K.: A characterization of locally homogeneous Riemann manifolds of dimension 3. Nagoya Math. J. 123 (1991), $77-90$.

Received 24.04.1997 